\title{
Multi-objective optimization of ultrasonic-assisted magnetic abrasive finishing process
}

\author{
Aviral Misra $^{1} \cdot$ Pulak M. Pandey $^{1}$ - U. S. Dixit ${ }^{2} \cdot$ Anish Roy $^{3} \cdot$ Vadim V. Silberschmidt $^{3}$
}

\begin{abstract}
Ultrasonic-assisted magnetic abrasive finishing (UAMAF) is an advanced abrasive finishing process that finishes a workpiece surface effectually when compared to a traditional magnetic abrasive finishing process in the order of nanometer. A change of surface roughness and material removal rate are two important factors determining the efficacy of the process. These two factors affect the surface quality and production time and, thereby, a total production cost. The finishing performed at higher material removal rates leads to a loss in shape/form accuracy of the surface. At the same time, increasing the rate of change of surface roughness increases loss of material. For an optimized finishing process, a compromise has to be made between the change of surface roughness and the material removal (loss). In this work, a multi-objective optimization technique based on genetic algorithm is used to optimize the finishing parameters in the UAMAF processes. A fuzzy-set-based strategy for a higher level decision is also discussed. The results of the optimization based on a mathematical model of the process are validated with the experimental results and are found to be in compliance.
\end{abstract}

Keywords UAMAF $\cdot$ Finishing $\cdot$ Multi-objective optimization $\cdot$ Genetic algorithm $\cdot$ Material removal $\cdot$ Surface roughness $\cdot$ Fuzzy sets

\section{Nomenclature}

A

Adj. MS

Amplitude of vibration

Adj. SS

Adjusted mean squares

$B H N$

$C$

$C_{T}$

$C_{R_{a}}$

$D_{a}$

$D_{i}$

$f$

$f_{e 1}$

Pulak M. Pandey

pmpandey@mech.iitd.ac.in

1 Department of Mechanical Engineering, Indian Institute of Technology Delhi, New Delhi 110016, India

2 Department of Mechanical Engineering, Indian Institute of Technology Guwahati, Guwahati 781039, India

3 Wolfson School of Mechanical, Electrical and Manufacturing Engineering, Loughborough University, Loughborough, Leicestershire LE11 3TU, UK $f_{e 2} \quad$ Objective function 2 of statistical model

$f_{m 1} \quad$ Objective function 1 of the model

$f_{m 2} \quad$ Objective function 2 of the model

FMAB Flexible magnetic abrasive brush

$g \quad$ Working gap

GA Genetic algorithm

$K_{s}$

$M_{a}$

$N$

$n_{a}$

$Q_{s}$

$Q_{T}$

$\% \Delta R_{a}$

$\% \Delta R_{\text {ath }}$

$\% \Delta R_{a e}$

RCSR

$R_{a 0}$

Steady-state material removal coefficient

Mesh size of abrasive particles

RPM of the electromagnet

Number of active abrasive particles

Steady-state mass material removal rate

Transient mass material removal

Percentage change in surface roughness value

Percentage change from mathematical model

Percentage change from statistical model

Rate of change of surface roughness

value

$R_{\text {acr }} \quad$ Critical surface roughness (minimum theoretical) value

$R_{a i}(T) \quad$ Instantaneous surface roughness value at time $T$.

$\mathrm{R}-\mathrm{S} \mathrm{q}$. Adjusted $R^{2}$

(Adj.) 


$\begin{array}{ll}T & \text { Finishing time } \\ t_{i} & \text { Depth of indentation } \\ \text { UMAPs } & \text { Unbonded magnetic abrasive particles } \\ V_{\text {avg }} & \begin{array}{l}\text { Average velocity of abrasive particle during } \\ \text { finishing }\end{array} \\ & \text { Supply voltage } \\ X_{1} & \text { Working gap } \\ X_{2} & \text { Percentage weight of abrasives } \\ X_{3} & \text { Mesh size of abrasive } \\ X_{4} & \text { Density of workpiece material } \\ \rho_{w} & \text { Angular velocity of abrasive particle } \\ \omega & \end{array}$

\section{Introduction}

Surface finish and dimensional accuracy are important for high-precision and high-accuracy components. In general, good surface finish is needed to improve corrosion resistance, fatigue strength, and esthetic appeal of a component. For a mating surface of a component, surface finish directly influences its tribological performance. A finishing operation is a crucial, expensive, difficult-to-control, and labour-intensive phase of the overall production and contributes significantly to the total production time and cost [1]. The requirement of a more precise surface finish increases the cost of finishing operations exponentially. The progress in this area has been made in automating the finishing operations to reduce the production time to some extent, but it increases the initial investment and operating costs significantly. The quality, cost, time, and performance of finishing operations can be improved considerably by choosing optimum values of the process parameters. The main objectives of any finishing process are to (i) minimize the final surface roughness value and (ii) minimize the size and shape inaccuracy [2]. To achieve the latter, the material loss during a finishing process should be as low as possible. The judicious selection of parameters not only improves the quality of the workpiece surface but also enhances the rate of change of surface roughness (RCSR) with very low material loss. This work aims at a multi-objective optimization of ultrasonic-assisted magnetic abrasive finishing (UAMAF) process to achieve these objectives.

An ultrasonic-assisted magnetic abrasive finishing (UAMAF) is an advanced abrasive finishing process that finishes a workpiece surface effectually when compared to a traditional magnetic abrasive finishing process in the order of nanometer. Hence, it contains some process similarities in terms of process mechanics as compared to MAF process. A schematic of an experimental set-up of the UAMAF process together with its photograph is shown in Fig. 1. In UAMAF, an electromagnet having alternatively arranged as North and South Pole was used. The mixture of ferromagnetic and abrasive particles is used as a finishing agent known as unbounded magnetic abrasive particles
(UMAPs). The UMAPs under the influence of magnetic field forms flexible magnetic abrasive brush (FMAB) [3]. The interaction between the FMAB and workpiece surface removes the micro-chipping as well as nano-scratching [3]. The UAMAF process when compared to the MAF process provides better results for similar set of processing parameters $[4,5]$. An improvement of $127 \%$ had been found for the change in surface roughness when finishing was performed at selected parameters [4].

Till now, to the authors' knowledge, only a single attempt was made to optimize the UAMAF process, by Mulik and Pandey [6]. They conducted experiments based on a response surface methodology to predict the percentage change in surface roughness and employed a Taguchi design of experiments to assess the total material removal. Regression equations obtained from the experimental data were converted into a single objective minimization function with varying weights. They used the "fmincon" function in an optimization toolbox of MATLAB® software to optimize the process parameters. The optimization results were validated with experimental observations.

In UAMAF, the magnitude of RCSR is a function of the material removal rate (MRR) [7]. To minimize the overall finishing time, one needs a higher RCSR. At the same time, to preserve the form of the surface as well as the size tolerance of the component, the MRR should be low. A high MRR requires a higher indentation of abrasive particles that leads to a higher magnitude of the critical (minimum achievable) surface roughness value [8]. Thus, a compromise has to be made between the MRR and the RCSR to obtain the good surface finish with a less time. In this work, the two conflicting objectives, i.e., the MRR and the percentage change of surface roughness, are optimized by using a multi-objective genetic algorithm. Such algorithms were conveniently used to optimize material removal processes with conflicting objectives [9-11]. Mathematical models developed by Misra et al. [7, 8] for material removal and surface roughness for UAMAF are used to estimate the two objective functions - total material removal and percentage change in surface roughness - in a given time. A Pareto front obtained by optimizing the theoretical model is compared with that obtained from optimization of statistical models developed with experiments. The paper presents three novel aspects, first, a method has been proposed to optimize the UAMAF process considering the theoretical models. Second, method has been proposed to optimize the UAMAF process using the multi-objective optimization (previously, only a single objective function has been used for the optimization). Lastly, paper presents an approach for a higher level of decision-making using Fuzzy sets. It uses the data obtained from the optimization for obtaining different optimized process parameters based on different weights assigned to the objective function. 
Fig. 1 Experimental setup of UAMAF. a Schematic diagram. b Actual system
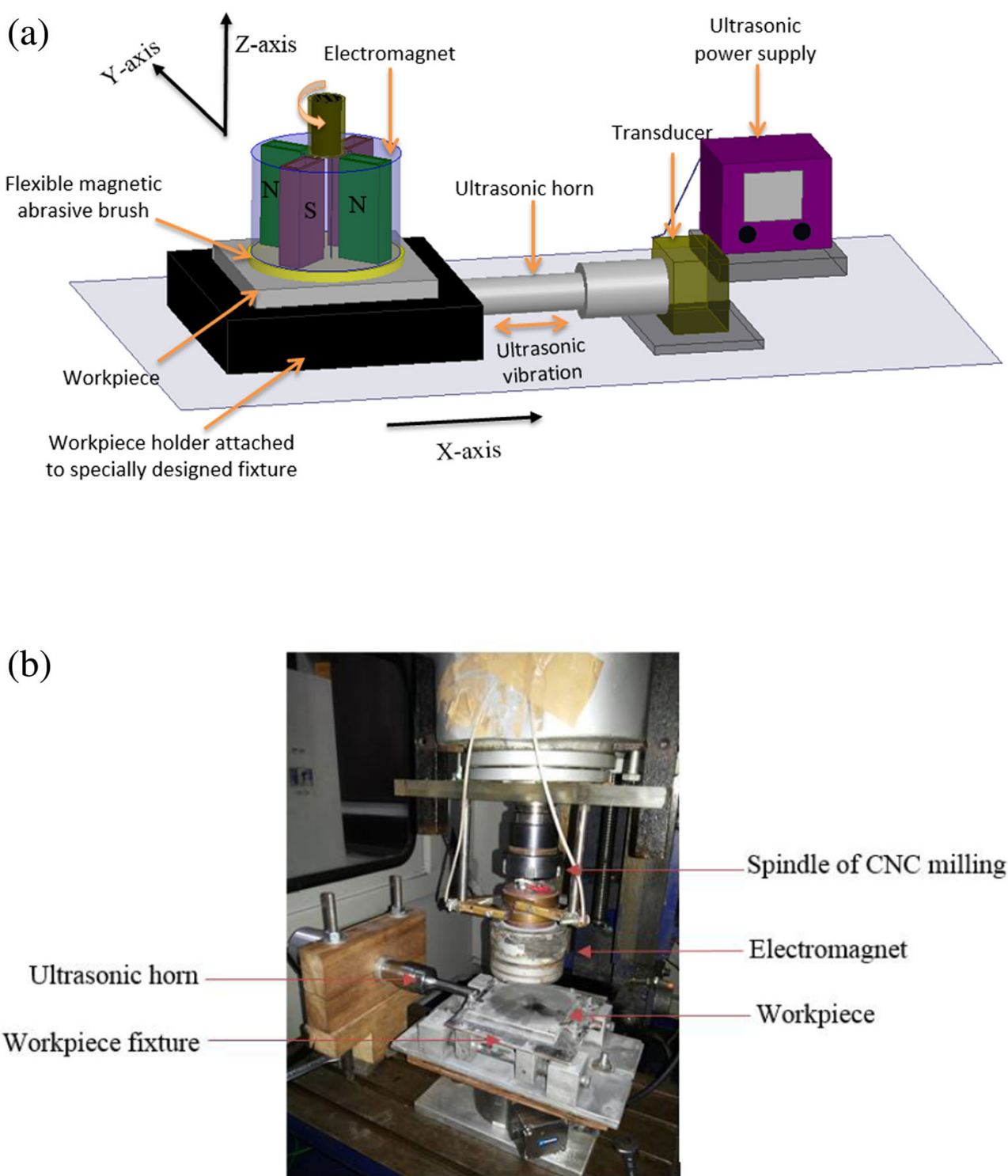

\section{Mechanics of UAMAF process}

The magnetic field produced by the electromagnet compels the ferromagnetic particles in UMAPs to align themselves along the magnetic lines due to dipolar interaction between them [12]; the non-magnetic abrasive particles in UMAPs are entrapped in space between the chains of ferromagnetic particles thus forming a flexible magnetic abrasive brush (FMAB) [13]. The strength of FMAB which acts as a multi-point cutting tool can be altered by varying the magnitude of supply voltage or current [14]. In the FMAB, only a few abrasive particles comes in contact with the workpiece surface and actually take part in the finishing action; they are termed as active abrasive particles. These particles experience a magnetic levitation force that enables them to create a microindentation onto the surface of the workpiece [15]. The rotation of electromagnet and the vibratory motion causes a relative motion between the active abrasive particles and the workpiece surface. Due to relative motion, the indented active abrasive particles actate an abrasion or scratching action on the surface of the workpiece (Fig. 2a). Apparently, the interaction of the workpiece surface with the FMAB originates contact stresses at the interaction points. Once these contact stresses exceed a critical value, irregularities are sheared-off from the workpiece surface (Fig. 2b). Thus, micro-chipping and nano-scratching are the phenomenon responsible for the material removal in UAMAF [16].

Various researchers $[5,17,18]$ had observed experimentally that initially during finishing, MRR is high, which decreases as the finishing proceeds and after a certain period of time, it becomes steady. Hence, during the modeling of UAMAF, it was assumed that at any instant, material removal consists of two simultaneous and independent phenomena - a steady-state and a transient removal phenomenon. The steady- 


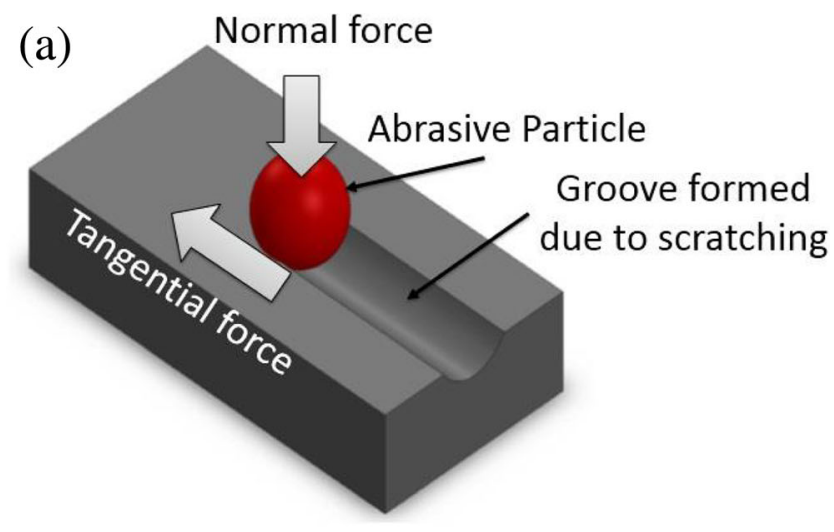

(b)

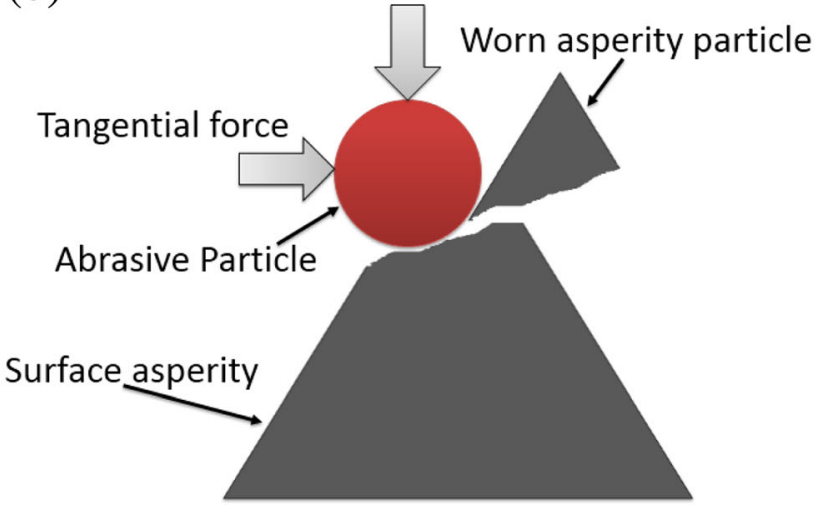

Fig. 2 Schematic of a nano-scratching and $\mathbf{b}$ micro-chipping

state phenomenon depicts the removal of material by scratching action of the abrasive, and it mainly depends upon the finishing condition (Fig. 2a). Mathematically, volumetric removal due to steady-state removal during the finishing is given by

$V_{\text {avg }}=K_{S} A_{H P} V_{\text {avg }} t$

where $K_{s}$ is steady-state material removal coefficient, $A_{H P}$ is the horizontal projected cross-sectional area of indentation, $V$ is the velocity of the abrasive particle, and $t$ is the finishing time. On the other hand, a transient volumetric removal is a function of the instantaneous amount of irregularities and it decreases with time. Mathematically,

$\frac{\mathrm{d} V_{T}}{\mathrm{~d} t} \propto V_{\text {iirr }}$

The summation of these two phenomena at any instant gives the instantaneous MRR. Also, the RCSR decreases with time. Thus, it had been assumed that it depends upon the instantaneous volume of surface irregularities on the workpiece and MRR. For the modeling purpose, it was assumed that RCSR is given by

$\frac{d R_{a}(t)}{d \tau} \propto\left\{R_{a_{i}}(t)-R_{a_{c r}}\right\} \dot{Q}$ where $Q$ is instantaneous material removal rate and $\left\{R_{a_{i}}(t)-R_{a_{c r}}\right\}$ represents the measure of volume of irregularities available for removal at the instant. Here, $R_{a_{c r}}$ denotes a "critical surface roughness value" beyond which further reduction in the value of surface roughness reduction is not possible and its magnitude depends upon the depth of indentation by the abrasive particle on the workpiece surface. Figure 3 illustrates the major finishing parameters that affect the finishing rate for UAMAF process.

\section{Multi-objective optimization}

The percentage change in surface roughness and total material removal of an UAMAF processed workpiece surface are considered as the objective functions to optimize the process. These two objectives are conflicting in nature. The formulation of the objective functions considered in the present work is described in the following subsections

\subsection{Percentage change in surface roughness}

A change in surface roughness value for a workpiece finished with the UAMAF process is calculated using the surface roughness model developed by Misra et al. [8]. According to the model, surface roughness in UAMAF is a function of hardness of workpiece material, the size distribution of UMAPs and process parameters, viz., supply voltage, working gap, rotational speed of the electromagnet, and amplitude and frequency of vibration. The expression proposed for surface roughness after the finishing time $T$ is given by [8]

$R_{a_{i}}(T)=\left\{R_{a_{0}}-R_{a_{c r}}\right\} \mathrm{e}^{\left[-C_{R_{a}} \cdot\left(Q_{s} T+Q_{T}\right)\right]}+R_{a_{c r}}$,

where $R_{a_{0}}$ is the initial surface roughness and $R_{a_{c r}}$ is the critical (minimum) surface roughness that can be obtained with the given process parameters. $Q_{s}$ is the steady state MRR given by [8]

$\dot{Q_{s}}=\frac{1}{4} K_{s} \rho_{w} n_{a}\left[D_{a}^{2} \sin ^{-1}\left(\frac{D_{i}}{D_{a}}\right)-D_{i}\left(D_{a}-t_{i}\right)\right] V_{\text {avg }}$

and $Q_{T}$ is the transient material removal after the finishing time $T$ and is given as [8]

$Q_{T}=2 R_{a 0} \rho_{w} A_{f}\left(1-e^{-C_{T} T}\right)$.

Here, $K_{s}$ is the steady-state coefficient, $C_{T}$ is the transient coefficient, and $C_{R_{a}}$ is the surface roughness coefficient, which are calculated by an inverse method. For details, one can refer to the article by Misra et al. [7]. The values of $K_{s}, C_{T}$, and $C_{R_{a}}$ were obtained as $4.60 \times 10^{-5}, 0.034$, and $3.289 \times$ $10^{-4}$, respectively [8]. The percentage change in surface roughness $\left(\% \Delta R_{a}\right)$ is defined as 


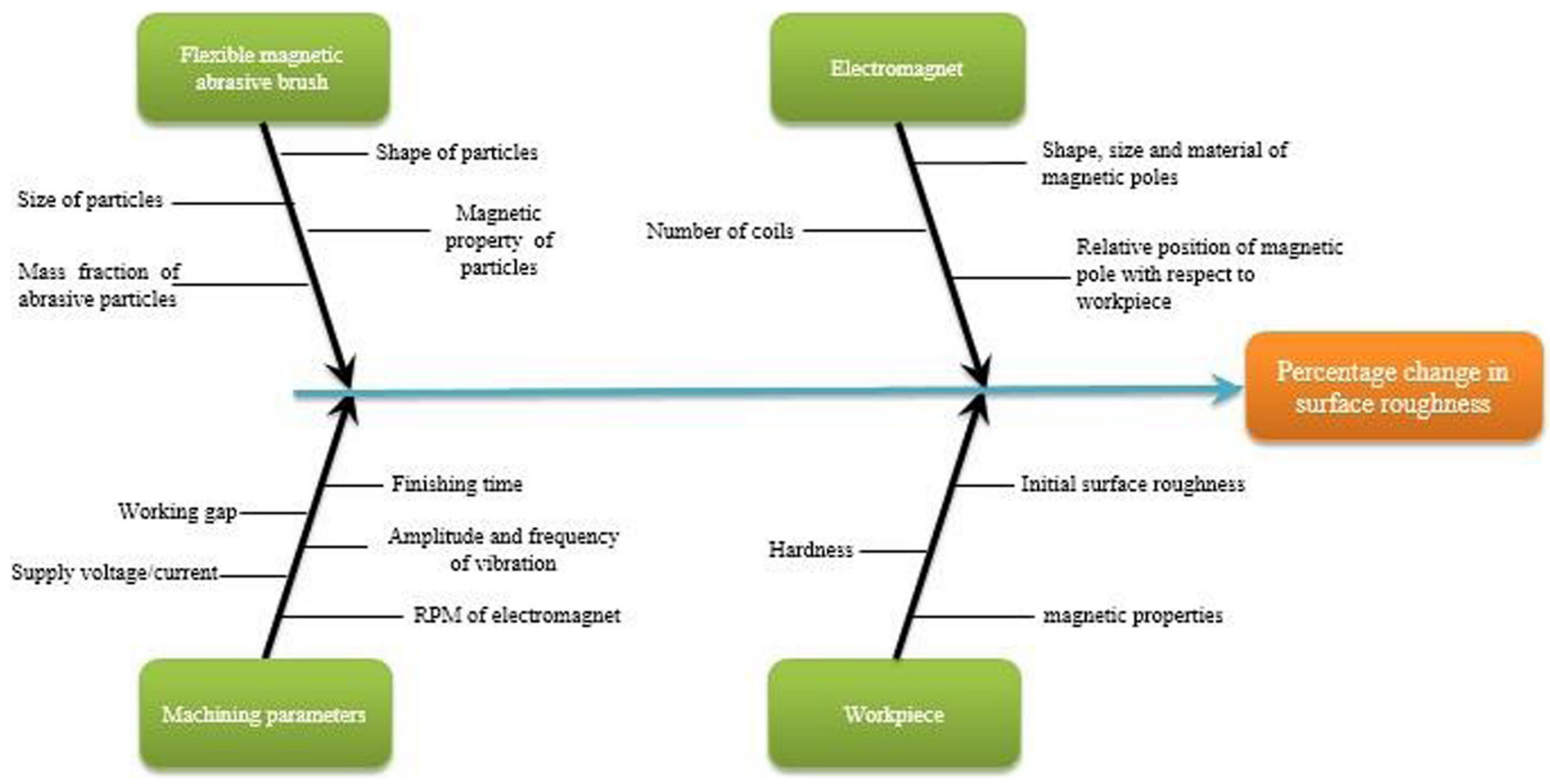

Fig. 3 Fishbone (Ishikawa) diagram showing the major finishing parameters for UAMAF process

$$
\begin{aligned}
\% \Delta R_{a}= & \frac{\text { Initial surface roughness }- \text { Final surface roughness }}{\text { Initial surface roughness }} \\
& \times 100 .
\end{aligned}
$$

Thus, the percentage change in surface roughness from the theoretical model $\left(\% \Delta R_{\text {ath }}\right)$ is given by

$\% \Delta R_{a t h}=\frac{R_{a_{0}}-R_{a_{i}}(T)}{R_{a_{0}}} \times 100$.

The final surface roughness value $R_{a_{i}}(T)$ after the finishing time $T$ in Eq. (8) is calculated from Eq. (4). The value of $\% \Delta R_{\text {ath }}$ should be maximized in order to minimize the final surface roughness value. Thus, one minimization objective function of the multi-objective optimization is

$$
f_{m 1}=\frac{1}{\% \Delta R_{a t h}} .
$$

\subsection{Total material removal}

The total material removal for achieving the prescribed value of surface roughness is an important factor, since it affects the form and accuracy of the finished surface. The material removal in UAMAF process at any instant is the result of two simultaneous and independent phenomena, i.e., a steady-state removal and a transient removal [7]. The material removal $(Q)$ after the finishing time $T$ after the start of the UAMAF process is given as
Table 1 Specification of options used in genetic algorithm toolbox

\begin{tabular}{ll}
\hline Option & Specification \\
\hline $\begin{array}{l}\text { Population size } \\
\text { Creation function (for creating the population) }\end{array}$ & 200 \\
Selection (reproduction) method & $\begin{array}{c}\text { Constraint } \\
\text { dependent }\end{array}$ \\
Tournament size & Tournament \\
Crossover fraction & 2 \\
Mutation function & 0.8 \\
& Constraint \\
Crossover function & dependent \\
& Intermediate (with \\
Migration direction (movement of the best individuals from one subpopulation to & ratio 1) \\
another subpopulation during parallel processing) & Forward \\
\hline
\end{tabular}


Table 2 Numerical values for material properties and finishing parameters

\begin{tabular}{ll}
\hline Parameters & Specification \\
\hline Workpiece material & SS 304 \\
Workpiece hardness $(B H N)$ & $231 \mathrm{BHN}$ \\
Initial surface roughness & $0.3234 \mu \mathrm{m}$ \\
Size of ferromagnetic particle (mesh number) & 300 (average) \\
Amplitude of vibrations & $8 \mu \mathrm{m}$ \\
Frequency of vibrations & $20 \mathrm{kHz}$ \\
Rotational speed of electromagnet & $300 \mathrm{rpm}$ \\
Steady-state coefficient $\left(K_{s}\right)$ & $4.600 \times 10^{-5}$ \\
Transient coefficient $\left(C_{T}\right)$ & 0.034 \\
Surface roughness constant $\left(C_{R_{a}}\right)$ & $3.289 \times 10^{-4}$ \\
Finishing time & $120 \mathrm{~s}$ \\
\hline
\end{tabular}

$Q=\dot{Q} T+Q_{T}$

where $Q_{S}$ and $Q_{T}$ are calculated from Eqs. (5) and (6), respectively. Thus, the second minimization objective function of the multi-objective optimization problem is

$f_{m 2}=Q$

The optimization problem can be stated as

Minimize $f_{m 1}$ and $f_{m 2}$

subject to

supply voltage : $40 \leq V \leq 80(\mathrm{~V})$, working gap : $1 \leq g \leq 2.5(\mathrm{~mm})$,

concentration, i.e., percentage of abrasive particles : $10 \leq C \leq 25$, mesh size of abrasive particles : $400 \leq M_{a} \leq 800$.

The percentage weight of abrasive is the weight of abrasive in the total weight of UMAPs (combined ferromagnetic and abrasive particles).

\section{Solution methodology}

The optimization objective is to select magnitudes of the process parameters such that during finishing the UAMAF process, both the surface roughness value and the material removal are minimized. The multi-model nature of this problem can be handled better using a genetic algorithm (GA) than a conventional optimization technique [9]. The GA is a powerful tool for solving multi-optimization problems [19]; it neither depends upon the initial solution nor considers any approximation. The GA is a meta-heuristic computerized search algorithm based on the principle of the natural selection and the natural genetics [20]. Initially, it begins with the origination of random solutions (termed as population). The generated solution is gauged for its fitness value; a higher fitness value signifies nobility of the solution. The population is then changed by basic operators of the GA - reproduction, crossover, and mutations - to create a new population. The new population is then weighed and verified for fulfilling the termination criterion. The entire procedure is repeated till a convergence is achieved. Performance of the GA is predominantly influenced by the population size, number of generations, crossover rate, and mutation rate [21].

The "optimization toolbox" in MATLAB R2015a was used to solve the formulated optimization problem (12)-(13). The solver used is "gamultiobj" - multi-objective optimization in the genetic algorithm [22]; parameters of the gamultiobj toolbox were set as per Table 1 . The objective functions given by Eqs. (9) and (11) were minimized within the variable bounds prescribed in Eq. (13). The constants of surface roughness and material removal models are given in Table 2 .
Table 3 Process parameters and levels for surface roughness and material removal in experiments

\begin{tabular}{lllll}
\hline Factors & & \multicolumn{2}{l}{ Levels } & \\
\cline { 3 - 5 } & & Low & Medium & High \\
\hline$X_{1}$ & Supply voltage (V) & 40 & 60 & 80 \\
$X_{2}$ & Working gap (mm) & 1.5 & 2 & 2.5 \\
$X_{3}$ & Percent weight of abrasive (\% wt) & 15 & 20 & 25 \\
$X_{4}$ & Mesh size of abrasive particles (sieve number) & 400 & 600 & 800 \\
\hline
\end{tabular}


Table 4 Experimental observation for percentage change in surface roughness and total material removal

\begin{tabular}{|c|c|c|c|c|c|c|}
\hline $\begin{array}{l}\text { Run } \\
\text { order }\end{array}$ & $\begin{array}{l}\text { Supply voltage } \\
\text { (in V) }\end{array}$ & $\begin{array}{l}\text { Working gap (in } \\
\mathrm{mm} \text { ) }\end{array}$ & $\begin{array}{l}\text { Percentage weight of } \\
\text { abrasive }\end{array}$ & $\begin{array}{l}\text { Mesh size of abrasives } \\
\text { (sieve no.) }\end{array}$ & $\begin{array}{l}\text { Percentage change in } R_{a} \\
\left(\% \Delta R_{a e}\right)\end{array}$ & $\begin{array}{l}\text { Total material removal } \\
(M R) \text { (in } \mathrm{mg})\end{array}$ \\
\hline 1 & 40 & 1.5 & 15 & 400 & 67.73 & 39.4 \\
\hline 2 & 40 & 2 & 20 & 600 & 59.98 & 28.7 \\
\hline 3 & 40 & 2.5 & 25 & 800 & 48.75 & 21.5 \\
\hline 4 & 60 & 1.5 & 20 & 800 & 68.70 & 42.5 \\
\hline 5 & 60 & 2 & 25 & 400 & 63.62 & 35.3 \\
\hline 6 & 60 & 2.5 & 15 & 600 & 63.72 & 31.5 \\
\hline 7 & 80 & 1.5 & 25 & 600 & 74.23 & 51.4 \\
\hline 8 & 80 & 2 & 15 & 800 & 68.96 & 46.0 \\
\hline 9 & 80 & 2.5 & 20 & 400 & 67.69 & 40.9 \\
\hline
\end{tabular}

\section{Validation}

In order to validate the multi-objective optimization model, the experiments were conducted based on the Taguchi's $\mathrm{L}_{9}$ (with 3 levels) orthogonal array design [23]. The process parameters and levels for experimentation are given in Table 3. The constant parameters used during experimentations are as follows: the rotational speed of electromagnet $300 \mathrm{rpm}$, the mesh size of ferromagnetic particles 300 (average), the amplitude of vibration $8 \mu \mathrm{m}$, and the frequency of vibration $20 \mathrm{kHz}$.

The initial value of surface roughness was measured by using a Talysurf (Taylor Hobson, Leicester, UK) with a Zheight resolution of $16 \mathrm{~nm}$ and a cut-off evaluation length of $0.8 \mathrm{~mm}$. The total material removed $(M R)$ (in $\mathrm{mg}$ ) was measured by weighing machine with the smallest measurement weight of $0.0001 \mathrm{~g}$ (A\&D Instruments India Private Limited Series GR-200). The details of the experimental design and obtained responses are given in Table 4.

The regression analysis was performed to formulate the equations obtained from the experimental data. The regression equation obtained for the percentage change in surface roughness $\left(\% \Delta R_{a e}\right)$ was

$$
\begin{aligned}
\% \Delta R_{a e}= & 83.47 \\
& +0.2868 X_{1}-10.17 X_{2}-0.460 X_{3}-0.01053 X_{4} .
\end{aligned}
$$

To check the goodness of fit for Eq. (14), an ANOVA analysis was performed; the results of this analysis for the percentage change in surface roughness are given in Table 5 . The value of R-Sq was found to be $94.83 \%$, which shows that Eq. (14) has a strong correlation with the experimental observation. The regression equation for the total material removed after the finishing based on the experimental response is given as

$M R=47.98$

$$
+0.4058 X_{1}-13.13 X_{2}-0.290 X_{3}-0.00467 X_{4} \text {. }
$$

The goodness of fit for Eq. (15) was also assessed with the ANOVA analysis; the R-Sq value was $97.70 \%$, demonstrating a strong correlation of Eq. (15) with the experimental observation for the material removal. The results of this ANOVA analysis are given in Table 6 .

The regression equations obtained were used to evaluate the objective functions in the multi-objective genetic algorithm tool-box in MATLAB $®$ to get a Pareto optimal front. Since, tool-box tends to minimize the objective functions, the objective functions used for multi-objective optimization are given as

Objective function 1:

$f_{e 1}=\frac{1}{\% \Delta R_{a e}}$,

Table 5 ANOVA for $\% \Delta R_{a e}$

\begin{tabular}{llllll}
\hline R-Sq (Adj) & \multicolumn{7}{l}{} \\
\hline Parameters & DOF & Adj. SS & Adj. MS & F-value & $p$ value \\
Supply voltage $\left(X_{1}\right)$ & 1 & 197.46 & 197.456 & 72.48 & 0.001 \\
Working gap $\left(X_{2}\right)$ & 1 & 155.04 & 155.042 & 56.91 & 0.002 \\
Percentage weight of abrasives $\left(X_{3}\right)$ & 1 & 31.79 & 31.786 & 11.67 & 0.027 \\
Mesh size of abrasives $\left(X_{4}\right)$ & 1 & 26.59 & 26.586 & 9.76 & 0.035 \\
Error & 4 & 10.90 & 2.724 & & \\
Total & 8 & 421.77 & & & \\
\hline
\end{tabular}




\begin{tabular}{llllll}
\hline R-Sq (Adj) & \multicolumn{7}{l}{} \\
\hline Parameters & DOF & Adj. SS & Adj. MS & F-value & $p$ value \\
Supply voltage $\left(X_{1}\right)$ & 1 & 395.282 & 395.282 & 196.41 & 0 \\
Working gap $\left(X_{2}\right)$ & 1 & 258.727 & 258.727 & 128.56 & 0 \\
Percentage weight of abrasives $\left(X_{3}\right)$ & 1 & 12.615 & 12.615 & 6.27 & 0.067 \\
Mesh size of abrasives $\left(X_{4}\right)$ & 1 & 5.227 & 5.227 & 2.60 & 0.182 \\
Error & 4 & 8.050 & 2.012 & & \\
Total & 8 & 679.9 & & & \\
\hline
\end{tabular}

Objective function 2:

$f_{e 2}=M R$.

The variable bounds used for the optimization were the same as given in Eq. (13).

The Pareto optimal fronts for both the model and the experimental data are presented in Fig. 4. It is clearly seen that the trend of both fronts is similar, with only a small quantitative difference between them. Thus, the optimization procedure based on the model was validated with the experimental observations and found to be in a good agreement. The trends show that as the objective function representing the inverse of percentage change in surface roughness increases, the second objective function, i.e., total material removal, decreases and vice versa. A higher-level decision is required to choose one solution from various available Pareto optimal solutions. For this purpose, a fuzzy-set-based methodology may be suitable as demonstrated in the next section.

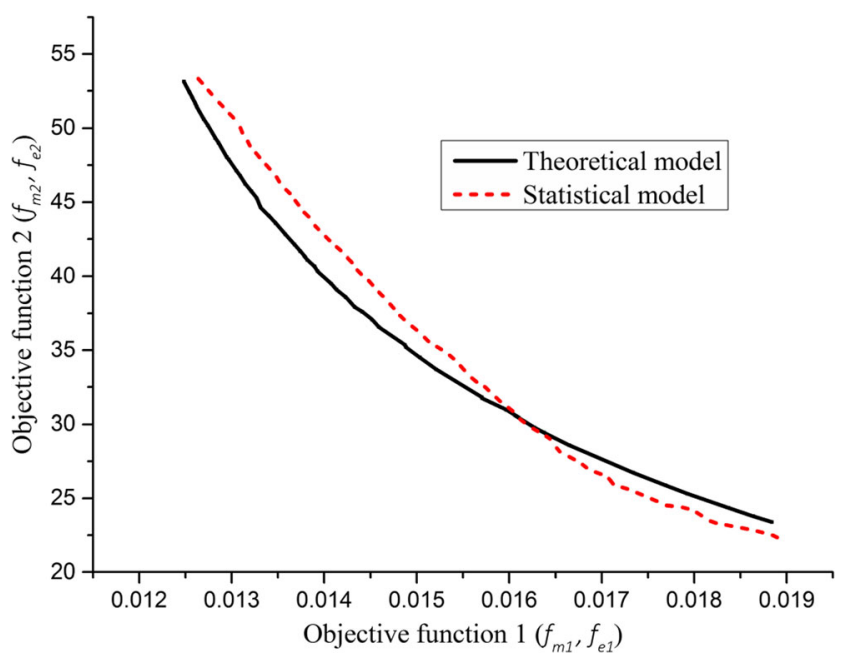

Fig. 4 Comparison of Pareto fronts obtained from theoretical and statistical models

\section{Fuzzy-set-based method for higher-level decision}

A higher-level decision to choose one solution from various possible Pareto solutions can be based on a fuzzy set theory [21, 24]. In this work, the two objectives are looked upon as fuzzy parameters. A membership grade $(\mu)$ is assigned for each solution, where 0 is assigned for the solution that manifest the least likely and 1 the most likely ones.

The strategy adopted for the construction of the membership function for the objective are as follows. In the Pareto optimal front, the lowest possible value of each objective was assigned a membership grade of 1 and the highest possible value was assigned a membership grade of 0.5 ; the membership grade varied linearly between these levels.

The different membership grades may be obtained for a particular solution considering different facet of the objective. The main aim of the fuzzy-set-based optimization procedure is to maximize the overall membership grade that denotes the overall preference of the customer. The two types of strategies were applied for obtaining the overall membership grade $\left(\mu_{0}\right)$, (a) a compensating trade-off strategy, where fully compensating trade-off function used is $\mu_{0}=\sqrt{\mu_{1} \mu_{2}}$ and (b) a noncompensating trade-off strategy, where fully noncompensating trade-off function is $\mu_{0}=\min \left[\mu_{1}, \mu_{2}\right]$, where $\mu_{1}$ and $\mu_{2}$ are the membership grades corresponding to two objectives respectively.

The overall membership grade is defined as the combination of these two strategies and is given as

$\mu_{0}=\alpha \sqrt{\left(\mu_{1} \mu_{2}\right)}+(1-\alpha) \min \left[\mu_{1}, \mu_{2}\right]$

The above equation reduces to a pure compensating tradeoff strategy for $\alpha=1$ and the pure non-compensating trade-off strategy for $\alpha=0$. A middle approach may be to take $\alpha=0.5$.

Table 7 depicts some Pareto optimal solutions obtained by solving optimization problems based on the model of the UAMAF process. It brings out many interesting features of multi-objective and multiple-optima problems. For example, following the compensating strategy, there are two best 
Table 7 Comparison of some Pareto optimal solutions based on model

\begin{tabular}{|c|c|c|c|c|c|c|}
\hline \multirow[t]{2}{*}{ Solution } & \multirow[t]{2}{*}{ Design variables $\left(X_{1}, X_{2}, X_{3}, X_{4}\right)$} & \multicolumn{5}{|c|}{ Membership grade } \\
\hline & & $\mu_{1}$ & $\mu_{2}$ & $\min \left[\mu_{1}, \mu_{2}\right]$ & $\sqrt{\left(\mu_{1} \mu_{2}\right)}$ & $\begin{array}{l}\text { Overall } \\
(\alpha=0.5)\end{array}$ \\
\hline 1 & $(74 \mathrm{~V}, 1.51 \mathrm{~mm}, 16 \%, 463)$ & 1 & 0.5 & 0.5 & 0.707 & 0.604 \\
\hline 2 & $(62 \mathrm{~V}, 1.56 \mathrm{~mm}, 18 \%, 532)$ & 0.832 & 0.729 & 0.729 & 0.779 & 0.754 \\
\hline 3 & $(67 \mathrm{~V}, 1.71 \mathrm{~mm}, 19 \%, 608)$ & 0.786 & 0.778 & 0.778 & 0.782 & 0.780 \\
\hline 4 & $(60 \mathrm{~V}, 1.76 \mathrm{~mm}, 23 \%, 512)$ & 0.762 & 0.799 & 0.762 & 0.780 & 0.771 \\
\hline 5 & $(59 \mathrm{~V}, 1.56 \mathrm{~mm}, 22 \%, 528)$ & 0.792 & 0.770 & 0.770 & 0.781 & 0.776 \\
\hline 6 & $(71 \mathrm{~V}, 1.77 \mathrm{~mm}, 17 \%, 613)$ & 0.809 & 0.756 & 0.756 & 0.782 & 0.769 \\
\hline 7 & $(46 \mathrm{~V}, 2.5 \mathrm{~mm}, 25 \%, 779)$ & 0.5 & 1 & 0.5 & 0.707 & 0.604 \\
\hline
\end{tabular}

solutions - solution 3 and solution 6. Both of them provide the membership grade equal to 0.782 but have different optimum design variables. However, following the non-compensating strategy, the best solution is solution 3 only. Providing equal weightage to the compensating and non-compensating strategies, i.e., for $\alpha=0.5$, the best solution is solution 3 with the overall membership grade of 0.780 . In fact, if a solution is the best from both compensating and non-compensating points of view, it will be the best solution for any chosen value of $\alpha$. In this problem, it is the case with solution 3 . For it, the percentage reduction in surface roughness is $68.5 \%$ and the material loss is $36.6 \mathrm{mg}$. At the design parameters of solution 3 , regression equations provided the reduction in surface roughness as $70.1 \%$ and material loss as $44.3 \mathrm{mg}$. These values are very close to those obtained from the model. Hence, the modeling and optimization are further validated.

There may be situations when both criteria may not be equally important. For example, if the main focus is on getting good surface finish alone, then solution 1 must be chosen because it provides the membership grade 1 for objective 1 . To account for varying importance of the two objectives, the overall membership grade may be defined as

$\mu_{0}=\alpha \times \sqrt{\left(\mu_{1}^{a} \mu_{1}^{b}\right)}+(1-\alpha) \times \min \left[\mu_{1}^{a}, \mu_{1}^{b}\right]$,

where $a$ and $b$ represent the weights of the two objectives. These can be decided by the experts. The literature contains some guidelines for fine-tuning the membership grades and deciding weights of the objectives based on subjective opinions of the experts [25].

\section{Conclusion}

In this work, an optimization model for the UAMAF process was developed. The two conflicting objectives, viz., the percentage change in surface roughness and the total material removal, were optimized using the multi-objective genetic algorithm. The developed model was validated by experimental observations. The following conclusions can be derived from the optimization model:

- The Pareto optimal front obtained from optimization of the presented model showed a good agreement with that obtained from the statistical model employing the experimental data.

- A fuzzy-set-based higher-level decision suggested a solution that provided $68.5 \%$ reduction in the surface roughness with a loss of only $36.6 \mathrm{mg}$ material. At that solution, the statistical model based on the experimental data predicts $70.1 \%$ of improvement in surface roughness and $44.3 \mathrm{mg}$ of material removal. This depicts a strong agreement of the employed model-based optimization procedure with the experiments.

\section{References}

1. Rao RV (2011) Advanced modeling and optimization of manufacturing processes. Springer, London

2. Jain NK, Jain VK, Jha S (2006) Parametric optimization of advanced fine-finishing processes. Int J Adv Manuf Technol 34: 1191-1213. https://doi.org/10.1007/s00170-006-0682-4

3. Verma GC, Kala P, Pandey PM (2017) Experimental investigations into internal magnetic abrasive finishing of pipes. Int J Adv Manuf Technol 88:1657-1668. https://doi.org/10.1007/s00170-016-88810

4. Mulik RS, Pandey PM (2011) Ultrasonic assisted magnetic abrasive finishing of hardened AISI 52100 steel using unbonded SiC abrasives. Int J Refract Met Hard Mater 29:68-77. https://doi.org/10. 1007/s00170-010-3102-8 
5. Yun H, Han B, Chen Y, Liao M (2016) Internal finishing process of alumina ceramic tubes by ultrasonic-assisted magnetic abrasive finishing. Int J Adv Manuf Technol 85:727-734. https://doi.org/ 10.1007/s00170-015-7927-z

6. Mulik RS, Pandey PM (2011) Experimental investigations and optimization of ultrasonic assisted magnetic abrasive finishing process. Proc Inst Mech Eng B J Eng Manuf 225:1347-1362. https:// doi.org/10.1177/09544054JEM2122

7. Misra A, Pandey PM, Dixit US (2017) Modeling of material removal in ultrasonic assisted magnetic abrasive finishing process. Int J Mech Sci 131-132:853-867. https://doi.org/10.1016/j.ijmecsci. 2017.07.023

8. Misra A, Pandey PM, Dixit US (2017) Modeling and simulation of surface roughness in ultrasonic assisted magnetic abrasive finishing process. Int J Mech Sci 133:344-356. https://doi.org/10.1016/j. ijmecsci.2017.08.056

9. Pandey PM, Thrimurthulu K, Reddy NV (2004) Optimal part deposition orientation in FDM by using a multicriteria genetic algorithm. Int J Prod Res 42:4069-4089. https://doi.org/10.1080/ 00207540410001708470

10. Quiza Sardiñas R, Rivas Santana M, Alfonso Brindis E (2006) Genetic algorithm-based multi-objective optimization of cutting parameters in turning processes. Eng Appl Artif Intell 19:127133. https://doi.org/10.1016/j.engappai.2005.06.007

11. Kuriakose S, Shunmugam MS (2005) Multi-objective optimization of wire-electro discharge machining process by non-dominated sorting genetic algorithm. J Mater Process Technol 170:133-141. https://doi.org/10.1016/j.jmatprotec.2005.04.105

12. Das M, Jain VK, Ghoshdastidar PS (2007) Analysis of magnetorheological abrasive flow finishing (MRAFF) process. Int J Adv Manuf Technol 38:613-621. https://doi.org/10.1007/ s00170-007-1095-8

13. Jayswal SC, Jain VK, Dixit PM (2005) Modeling and simulation of magnetic abrasive finishing process. Int J Adv Manuf Technol 26: 477-490. https://doi.org/10.1007/s00170-004-2180-x
14. Singh DK, Jain VK, Raghuram V (2006) Experimental investigations into forces acting during a magnetic abrasive finishing process. Int J Adv Manuf Technol 30:652-662. https://doi.org/10. 1007/s00170-005-0118-6

15. Misra A, Pandey PM, Dixit US, Roy A, Silberschmidt VV (2017) Modeling of finishing force and torque in ultrasonic-assisted magnetic abrasive finishing process. Proc Inst Mech Eng B J Eng Manuf 0:95440541773757. https://doi.org/10.1177/0954405417737579

16. Mulik RS, Pandey PM (2010) Mechanism of surface finishing in ultrasonic-assisted magnetic abrasive finishing process. Mater Manuf Process 25:1418-1427. https://doi.org/10.1080/10426914. 2010.499580

17. Wang Y, Hu D (2005) Study on the inner surface finishing of tubing by magnetic abrasive finishing. Int J Mach Tools Manuf 45:43-49. https://doi.org/10.1016/j.ijmachtools.2004.06.014

18. Fox M, Agrawal K, Shinmura T, Komanduri R (1994) Magnetic abrasive finishing of rollers. CIRP Ann Manuf Technol 43:181184. https://doi.org/10.1016/S0007-8506(07)62191-X

19. Thrimurthulu K, Pandey PM, Reddy NV (2004) Optimum part deposition orientation in fused deposition modeling. Int $\mathrm{J}$ Mach Tools Manuf 44:585-594. https://doi.org/10.1016/j.ijmachtools. 2003.12.004

20. Deb K (2001) Multi-objective optimization using evolutionary algorithms. Wiley, Hoboken

21. Dixit PM, Dixit US (2008) Modeling of metal forming and machining processes : by finite element and soft computing methods. Springer-Verlag, London

22. MathWorks Global Optimization Toolbox User's Guide. MathWorks

23. Montgomery DC (2013) Design and analysis of experiments, 8 th edn. Wiley, Hoboken

24. Bojadziev G, Bojadziev M (1996) Fuzzy sets, fuzzy logic, applications. World scientific

25. Hazarika M, Dixit US (2015) Assigning and fine tuning of fuzzy membership grades. In: Setup planning for machining. Springer International Publishing, Cham, pp 91-110 